\title{
A simple lectin-based biochip might display the potential clinical value of glycomics in patients with spontaneous intracerebral hemorrhage
}

\author{
Lei Ye ${ }^{1 \#}$, Yong-Sheng Fang ${ }^{2 \#}$, Xiao-Xue $\mathrm{Li}^{3}$, Yi Gao ${ }^{4}$, Sheng-Sheng Liu ${ }^{4}$, Qiang Chen ${ }^{4}$, Qiang $\mathrm{Wu}^{2}$, \\ Hong-Wei Cheng', Wei-Dong Du ${ }^{4}$
}

${ }^{1}$ Department of Neurosurgery, the First Affiliated Hospital of Anhui Medical University, Hefei, China; ${ }^{2}$ Department of Pathology, the Second Affiliated Hospital of Anhui Medical University, Hefei, China; ${ }^{3}$ Institute of Pathology, University Medical Center Göttingen, Göttingen, Germany; ${ }^{4}$ Department of Pathology, Anhui Medical University, Hefei, China

Contributions: (I) Conception and design: L Ye, YS Fang, WD Du; (II) Administrative support: WD Du, HW Cheng, Q Wu; (III) Provision of study materials or patients: L Ye, HW Cheng; (IV) Collection and assembly of data: Y Gao, Q Chen, XX Li; (V) Data analysis and interpretation: SS Liu, XX Li; (VI) Manuscript writing: All authors; (VII) Final approval of manuscript: All authors.

\#These authors contributed equally to this work.

Correspondence to: Wei-Dong Du. Department of Pathology, Anhui Medical University, Meishan Road 81, Hefei 230032, China. Email: weidong.du@ahmu.edu.cn; Hong-Wei Cheng. Department of Neurosurgery, the First Affiliated Hospital of Anhui Medical University, Jixi Road 218, Hefei 230022, China. Email: hongwei.cheng@ahmu.edu.cn; Qiang Wu. Department of Pathology. Second Affiliated Hospital of Anhui Medical University, Furong Road 678, Hefei 230601, China. Email: wuqiang@ahmu.edu.cn.

Background: Intracerebral hemorrhage $(\mathrm{ICH})$ is a cerebrovascular disease with extremely high disability and mortality rates. Glycans play critical roles in biological processes. However, whether glycans can serve as potential biomarkers for determining clinical diagnosis and prognosis in ICH remains determined.

Methods: In this study, we established a lectin-biochip to measure serum glycans levels in ICH patients $(n=48)$ and healthy controls $(n=16)$. An enzyme-linked immunosorbent assay (ELISA) was carried out to determine serum levels of IL-10 and TNF- $\alpha$ in the patients. Correlation analyses of the serum glycan and cytokine levels and the clinicopathological parameters of patients were performed.

Results: The biochip-based data revealed that the serum levels of $\alpha-M a n / \alpha-G l c$ (ConA), Gal $\beta 3$ GalNAc (PNA), GalNAc (VVA), Fuc $\alpha 6$ GlcNAc (AAL), $\alpha$-Fuc (LTL), and Gal $\beta 3$ GalNAc-Ser/Thr (AIL) significantly increased in the super-acute phase of ICH in comparison with healthy controls. Clinicopathological analysis indicated the serum levels of ConA, VVA, and LTL had significant associations with the National Institute of Health Stroke Scale (NIHSS), and serum VVA levels had a significant association with the Mini-Mental State Examination (MMSE) at day 90 after ICH. Correlation coefficient analysis revealed significant correlations between TNF- $\alpha$ and ConA $(\mathrm{P}<0.001)$ as well as between IL-10 and ConA $(\mathrm{P}<0.001), \mathrm{PNA}(\mathrm{P}=0.02)$, VVA $(\mathrm{P}<0.001)$, and MAL $(\mathrm{P}=0.04)$, respectively.

Conclusions: We established a proof-of-concept platform for detecting serum glycomics and highlighted their potential value in diagnosing and predicting ICH patients' outcomes.

Keywords: Spontaneous intracerebral hemorrhage (spontaneous ICH); glycomics; diagnosis; prognosis; inflammation

Submitted Nov 06, 2020. Accepted for publication Jan 18, 2021.

doi: $10.21037 / \mathrm{atm}-20-7315$

View this article at: http://dx.doi.org/10.21037/atm-20-7315 


\section{Introduction}

Intracerebral hemorrhage $(\mathrm{ICH})$ is a devastating cerebrovascular disease of the central nervous system (CNS), which results from the rupture of intracranial vessels with pathological lesions causing blood to break into the surrounding brain tissues (1). Although ICH's morbidity accounts for only $10-15 \%$ of all stroke patients, the 1 -month fatality can be as high as $38.5 \%$ (2). The occurrence of stroke is often recognized by those affected or family members and is subsequently validated by computed tomography (CT) or CT angiography (CTA) scans in the emergency department (ED), where clinical data is assessed by specialist neurologists (3). The early diagnosis of stroke is essential, but this is challenged by a lack of fast, specific, and sensitive detection approaches. Therefore, auxiliary diagnostic methodologies are urgently needed in clinical practice.

There are various potential molecule biomarkers available for recognizing ICH, providing multiple clinical or laboratory targets for diagnosis and prediction of outcomes in ICH patients (4,5). Promising biomarkers for the assessment of brain injury include $S 100 \beta$ and neuron-specific enolase (NSE), and these are used in the investigation of diverse neurocritical pathologies (6). Other biomarkers, including CD163 (7) and glial fibrillary acidic protein (GFAP) (8), have been suggested as candidate molecules for ICH diagnosis. However, most biomarker molecules are proteins or RNAs, and their expression levels are influenced not only by the status of the disease but also by genetic factors. Perplexing heterogeneous manifestations in clinical chemical measurements often lead to interpretation biases in diseases.

However, glycans are carbohydrate-based polymers and act as essential biomolecules in living organisms. The lack of a direct genetic template or structure of glycosylation, which itself ubiquitously occurs in post-translational modifications, means glycans are predominantly affected by micro-environmental factors in the body or the disease itself (9). Protein glycosylation played a pivotal role in numerous biological processes, including cell adhesion, molecule trafficking, pathogen recognition, clearance, signal transduction, and endocytosis (10) and a recent study showed the protein glycosylation was significantly correlated to the pathogenesis of stroke. Liu et al. reported that the increased level of bisecting GlcNAc and decreased level of sialylation and galactosylation might enhance the probability of ischemic stroke (IS) (10). In a serum glycoproteomic study, Deng et al. reported that both ConArelated and WGA-related glycoproteins were significantly correlated with favorable neurological outcomes in hypothermia-treated cardiac arrest patients, serving as potentially predicting biomarkers (11). Investigations of glycans' metabolism revealed fruitful evidence that the identification of glycosylation composition might provide a novel insight into the pathogenesis of CNS disease. However, most previous omic-related studies in stroke were carried out at the genetic and protein levels, and little research on $\mathrm{ICH}$ has focused on glycomics.

In this study, we presented a lectin-biochip method for the fast detection of serum glycomics profiles, preliminarily exploring the potential diagnostic and prognostic value of glycans in $\mathrm{ICH}$. We also performed a correlation investigation on the serum levels of glycans and neuroinflammatory responses in patients with ICH. We present the following article in accordance with the STARD reporting checklist (available at http://dx.doi.org/10.21037/ atm-20-7315).

\section{Methods}

\section{Patients}

In this study, 48 patients with ICH and 16 healthy controls with gender- and age-match were randomly recruited from the First Affiliated Hospital of Anhui Medical University between January 2017 and July 2018. Unrelated and genderand age-matched healthy controls were recruited from the medical examination center of the hospital. Healthy subjects who suffered from CNS diseases, autoimmune diseases, and malignant cancers were excluded from the study. Demographic data are summarized in Table 1. Two senior neurosurgeons conducted the clinical diagnosis of ICH according to symptoms and medical imaging results. Sera from each patient were collected at the identical time point of day 0 (the day at admission and within the 4 hours of disease onset). Sera were also collected from the control individuals at identical times. The sera of both groups were stored at $-80{ }^{\circ} \mathrm{C}$. All patients received additional clinical examinations to assess neurological damage based on the Glasgow Coma Scale (GCS) at admission.

Furthermore, both the National Institute of Health Stroke Scale (NIHSS) and Mini-Mental State Examination (MMSE) were used to assess the neural function status and cognitive function recoveries at day 90 after ICH operation. Two experienced senior neurologists provided evaluation for 
Table 1 Demographic data for the participants

\begin{tabular}{lccc}
\hline General information & ICH patients $(\mathrm{n}=48)$ & Healthy subjects $(\mathrm{n}=16)$ & $\mathrm{P}$ value \\
\hline Age & $61.25 \pm 5.91$ & $59.95 \pm 3.11$ & 0.202 \\
Gender, male & 31 & 10 & 0.880 \\
Hypertension & 34 & 9 & 0.282 \\
Diabetes mellitus & 7 & 4 & 0.339 \\
Dyslipidemia & 10 & 4 & 0.727 \\
\hline
\end{tabular}

$\mathrm{ICH}$, intracerebral hemorrhage.

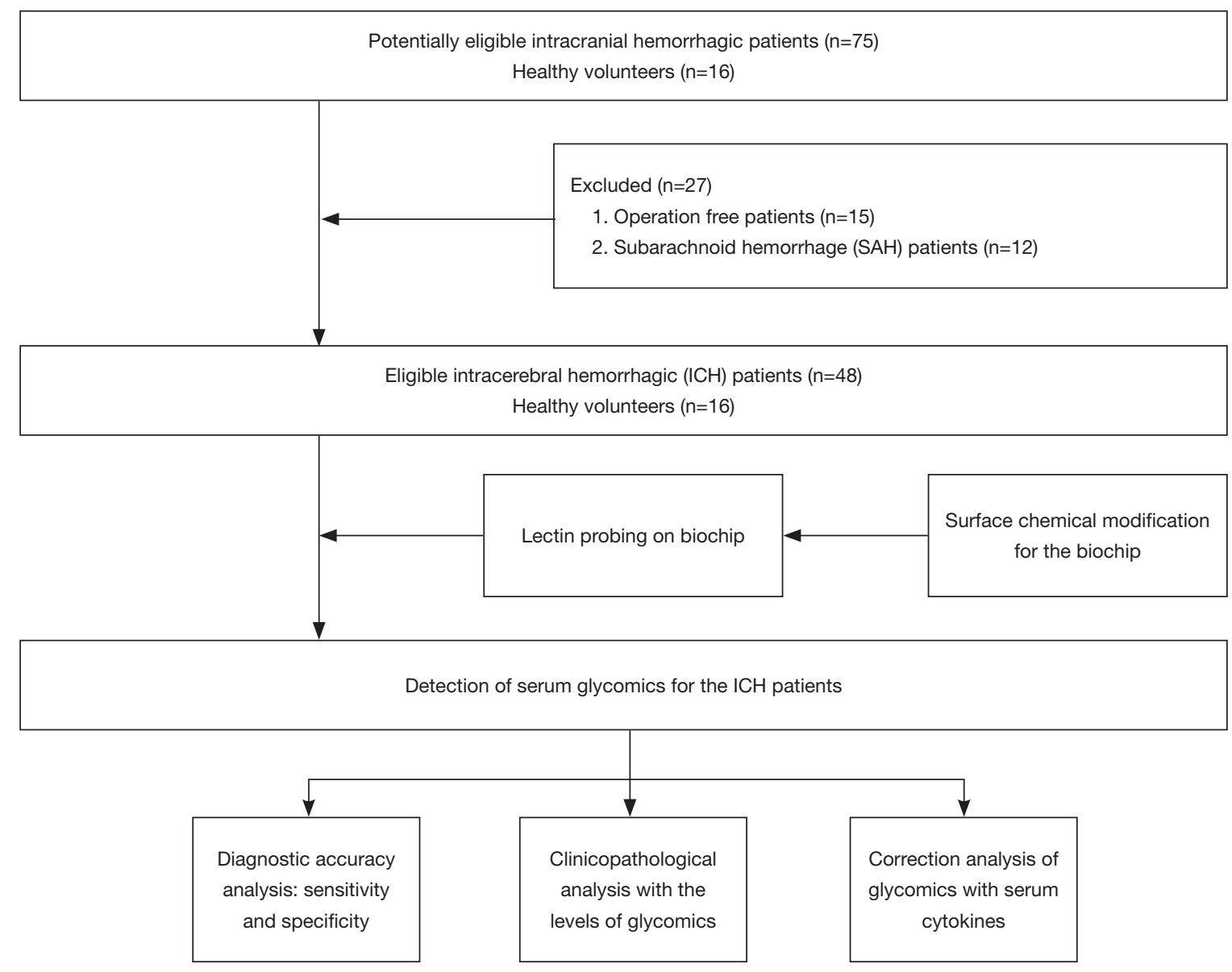

Figure 1 Flow diagram of the study.

scoring, and the flow diagram is depicted in Figure 1. The study was conducted following the Declaration of Helsinki (as revised in 2013) and was approved by the regional ethics board of the First Affiliated Hospital of Anhui Medical University (No. P2020-12-07). Informed consent was obtained from all individual participants.

\section{Reagents and instruments}

All chemicals were reagent grade and used as received 
unless indicated otherwise. Gold biochips were generously supplied by the Interactive Division, Thermo-Hybaid, Ulm, Germany. The geometric dimension of the biochip is described elsewhere. Gold film $(0.1 \mu \mathrm{m})$ with an initial adhesion layer of Teflon $(50 \mu \mathrm{m})$ was coated on glass slides $(75 \mathrm{~mm} \times 25 \mathrm{~mm})$, and the Teflon film divided the gold surface into a matrix spot layout of $8 \times 24$. Each spot diameter was $1.5 \mathrm{~mm}$. Lectins, including ConA, PNA, VVA, MAL-I, AAL, TLT, NPL, SNA, RCA-I, and AIL, were commercially obtained from Vector Laboratories Inc. (CA, USA). We purchased Cy3 from AAT Bioquest (CA, USA), and a BCA protein assay kit was obtained from Beyotime Biotechnology (Shanghai, China). A BSA, HEPES buffer, and PBST buffer were purchased from Sigman-Alerich (MO, USA), and a Proteo Prep immunoaffinity Albumin and IgG Depletion Kit was used as an isolation medium of albumin and $\mathrm{IgG}$ from the sera. For the removal of redundant cy3, PD MiniTrap G-25 chromatographic columns were obtained from GE (MA, USA), and an enzyme-linked immunosorbent assay (ELISA) kit for testing TNF- $\alpha$ and IL-10 was obtained from Elabscience (Wuhan, China). A Luxscan ${ }^{\mathrm{TM}}$ 10K-A microscanner was used (Capitalbio Co., Ltd., Beijing, China), and the microplate reader was a Bioreader (Model 550, Japan).

Chemical modification of the gold biochip was carried out according to a previously established approach (12). All reagents for the surface chemical modification were commercially obtained and included 16-amino-1-hexadecanethiol (16-AHDT, Dojindo, Shanghai, China), N-Succinimidyl 4-(maleimidomethyl) cyclohexanecarboxylate (SMCC, Sigma-Aldrich, MO, USA), 4-(dimethylamino) pyridine (DMAP, Sigma-Aldrich, MO USA), and N, N-Dimethylformamide (DMF, 99\% pure, Sigma-Aldrich, MO USA). The physical and chemical features and the biocompatibility of the modified surface were proven to be appropriate for bio-molecule probing (12).

\section{Purification of and preparation of Cy3-labeling sera}

Isolation of high-abundant proteins was introduced in our previous study (13). According to the manufacturer's protocol, high-abundant proteins, including albumin and IgG, were filtrated with a Proteo Prep Immunoaffinity Albumin and IgG Depletion Kit. Briefly, chromatographic columns were pretreated with equilibrium solution, then $40 \mu \mathrm{L}$ of the sera were individually diluted up to a volume of $100 \mu \mathrm{L}$ with the equilibrium solution. The diluted solution was added to a purification column and incubated for $10 \mathrm{~min}$ at room temperature (RT); then, centrifugation of the purification column was carried out at $10,000 \mathrm{rpm}$ at $4{ }^{\circ} \mathrm{C}$ for 60 seconds. The equilibrium solution was again added into the column and then incubated for $10 \mathrm{~min}$. Sera without high-abundant proteins were collected after centrifugation at $10,000 \mathrm{rpm}$ at $4{ }^{\circ} \mathrm{C}$ for $1 \mathrm{~min}$. Finally, a BCA Protein Assay kit was used for quantification of the serum proteins.

Preparation for $\mathrm{Cy} 3$ labeling sera was also introduced in our previous study (13). The purified sera described above were diluted with $0.01 \mathrm{M}$ PBST $(\mathrm{pH}$ 7.4) in a ratio of 1:5. The diluted sera were then incubated with an equivalent volume of Cy3-NHS (V) and $0.1 \mathrm{M}$ sodium bicarbonate buffer $(\mathrm{pH} 8.5)$ in a dark environment at RT for $1 \mathrm{~h}$, to attach the $\mathrm{Cy} 3$ tag to the purified sera. Removal of redundant Cy3 was performed with a PD MiniTrap G-25 column, and finally, Cy3-labelled sera were obtained.

\section{Lectin probing on biochips}

Before probing lectins on biochips, the chemically modified biochips were rinsed with PBST buffer and dried with nitrogen flow. Lectins, including ConA, PNA, VVA, MAL-I, AAL, TLT, NPL, SNA, RCA-I, and AIL, were individually resolved in $10 \mathrm{mM}$ HEPES buffer ( $\mathrm{pH}$ 8.5)$0.001 \%$ BSA to the concentration of $1 \mathrm{mg} / \mathrm{mL}$. Lectins were then immobilized on individual spots on the biochip surface and incubated at RT for 2 hours. The layout of the biochip is depicted in Figure $2 A$. The biochips were then washed with a $0.01 \mathrm{M}$ PBST buffer ( $\mathrm{pH}$ 7.4) and dried with a nitrogen flow.

\section{Profiling glycosylation of sera in ICH}

The Cy3-labelled sera from $48 \mathrm{ICH}$ patients and 16 healthy controls were supplied on the lectin-probed biochips and incubated in a humidity chamber and dark environment at RT for 0.5 hours. After being rinsed in PBST buffer twice at RT for $3 \mathrm{~min}$ and dried with a flow of nitrogen, the biochips were scanned with the microarray scanner. The fluorescence intensity on the biochips was recorded.

\section{ELISA for measurement of serum TNF- $\alpha$ and IL-10 in} $\mathrm{ICH}$

According to the manufacturer's protocols and based on the quantitative sandwich enzyme immunoassay technique, 
A

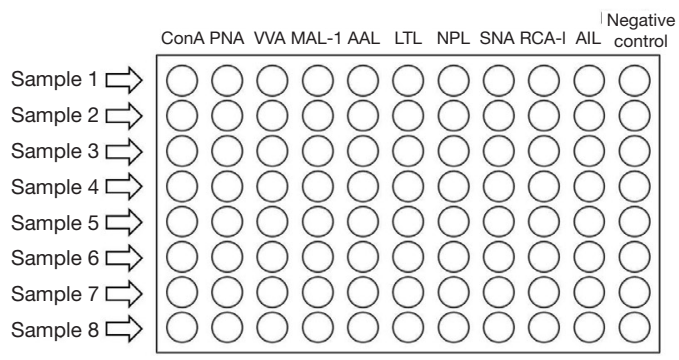

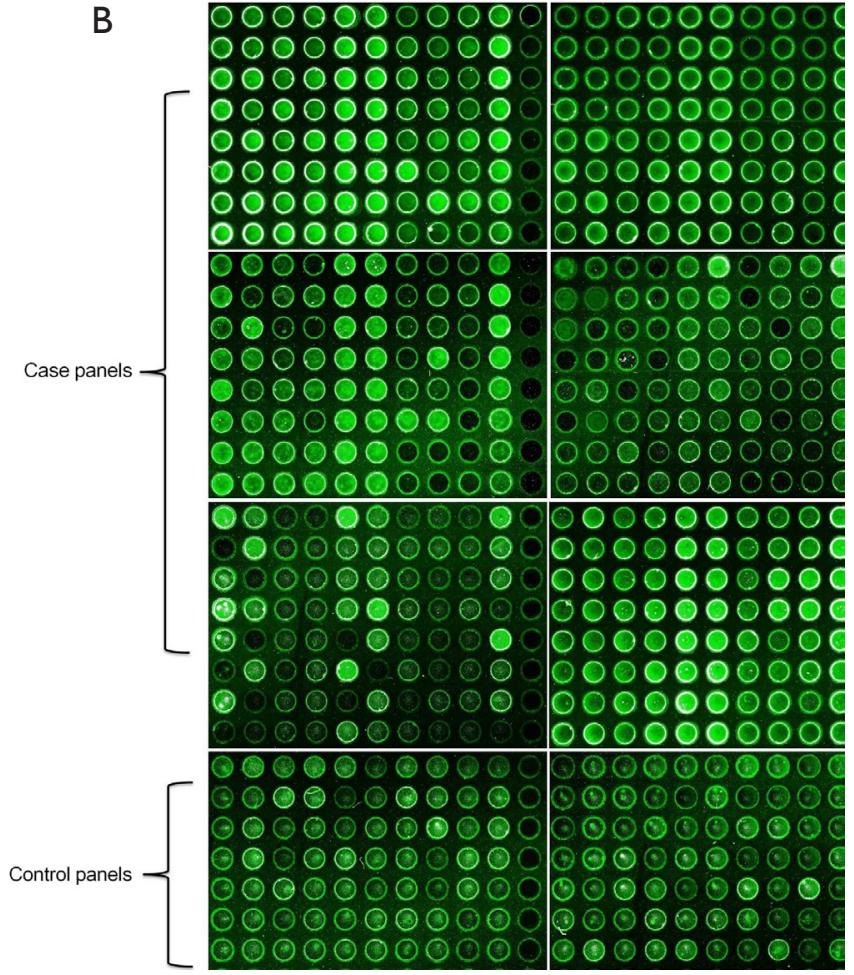

Figure 2 Biochip detection for the serum glycomics. (A) Layout of the biochip format. (B) Lectin-based biochip assay for measurement of serum glycans in $48 \mathrm{ICH}$ patients and 16 healthy controls. $\mathrm{ICH}$, intracerebral hemorrhage.

serum levels of TNF- $\alpha$ and IL-10 in the ICH patients were measured with ELISA kits.

\section{Statistical analysis}

SPSS 19.0 software was applied for statistical analysis. All enumeration data were given as mean \pm standard deviation (mean $\pm \mathrm{SD}$ ) and analyzed by the Kruskal-Wallis test. Ranked data were expressed by the median [interquartile range (IQR)] and were statistically analyzed using the Mann-Whitney test, and the Chi-squared test analyzed binary data. Correlations were calculated using Spearman's correlation analysis. The $\mathrm{P}$ values reported in the study were based on a two-sided probability test with a significance level of $\mathrm{P}<0.05$.

\section{Results}

\section{Glycosylation profiles of sera}

In this study, a well-established lectin-loaded biochip was used for serum glycomics profiling in both ICH patients and healthy controls. Ten lectins and the specific binding glycans are listed in Table 2. Differences in the 10 serum glycans at admission between the ICH patients and the healthy controls are shown in Figure 2B. Obviously, in comparison with the healthy controls, statistically increased serum levels in ConA $(\alpha-M a n / \alpha-G l c)(P<0.001)$, PNA (Gal $\beta 3$ GalNAc) $(\mathrm{P}<0.001)$, VVA (GalNAc) $(\mathrm{P}=0.03)$, AAL (Fuca6GlcNAc) $(\mathrm{P}<0.001)$, LTL $(\alpha-\mathrm{Fuc})(\mathrm{P}<0.001)$, and AIL (Gal $\beta 3$ GalNAc-Ser/Thr) $(\mathrm{P}<0.001)$, respectively, were observed in the ICH patients (Figure 3). No differences were found in the MAL-I (Gal $\beta 4 G l c N A c)$, NPL ( $\alpha$-Man), SNA (Neu5Aca6Gal/GalNAc), and RCA-I (Gal) $(\mathrm{P}>0.05)$.

\section{Correlation study of serum levels of glycans and clinical features in ICH}

We analyzed clinicopathological features, including age, gender, hypertension, diabetes, dyslipidemia, GCS, NIHSS, and MMSE, bleeding, midline shift $>10 \mathrm{~mm}$, and intravascular hemorrhage (IVH), in the $\mathrm{ICH}$ 
patients with the differentially expressed serum glycans. As shown in Table 3, AIL (Galß3GalNAc-Ser/Thr) was significantly correlated with age $(\mathrm{P}<0.001)$, gender $(\mathrm{P}=0.02)$, hypertension $(\mathrm{P}<0.001)$, and IVH $(\mathrm{P}<0.001)$, respectively. Significant correlations were also seen between LTL $(\alpha$-Fuc) and dyslipidemia $(\mathrm{P}<0.001)$, GCS $(\mathrm{P}=0.02)$, and NIHSS ( $\mathrm{P}=0.02$ ), and AAL (Fuca6GlcNAc) had obvious correlations with gender $(\mathrm{P}=0.01)$, dyslipidemia $(\mathrm{P}=0.02)$, GCS $(\mathrm{P}<0.001)$, and bleeding $(\mathrm{P}=0.001)$. Also, VA (GalNAc) revealed a significant statistical correlation with GCS $(\mathrm{P}<0.001)$, NIHSS $(\mathrm{P}<0.001)$, and MMSE $(\mathrm{P}<0.001)$.

Table 2 Lectins and binding glycans

\begin{tabular}{|c|c|}
\hline Lectins & Relevant glycans \\
\hline ConA & $\alpha-M a n / \alpha-G \mid c$ \\
\hline PNA & Galß3GalNAc \\
\hline VVA & GalNAc \\
\hline MAL-I & Galp4GlcNAc \\
\hline AAL & Fuc $\alpha 6$ GlcNAc \\
\hline LTL & $\alpha-F u c$ \\
\hline NPL & $\alpha-\operatorname{Man}$ \\
\hline SNA & Neu5Aca6Gal/GalNAc \\
\hline RCA-I & Gal \\
\hline AIL (Jacalin) & Galß3GalNAc-Ser/Thr \\
\hline
\end{tabular}

Significant correlations were also observed in ConA ( $\alpha-M a n$, $\alpha$-Glc) with NIHSS $(\mathrm{P}=0.03)$ and PNA (Gal $\beta 3$ GalNAc) with IVH $(\mathrm{P}=0.001)$.

\section{Preliminary analysis of the diagnostic potential of serum glycan levels in ICH patients}

We found elevated serum levels relevant to six glycanrelated lectins (ConA, PNA, VVA, AAL, LTL, and AIL) in the $48 \mathrm{ICH}$ patients. We completed a preliminary evaluation of the glycans' diagnostic potential using a receiver operating characteristic (ROC) curve (Figure S1). The area under curve (AUC) were yielded as 0.93 for ConA $(\mathrm{P}<0.01), 0.95$ for PNA $(\mathrm{P}<0.01), 0.67$ for VVA $(\mathrm{P}=0.04)$, 0.92 for AAL $(\mathrm{P}<0.01), 0.86$ for LTL $(\mathrm{P}<0.01)$, and 0.84 for AIL $(\mathrm{P}<0.01)$, respectively. We found that the sensitivity and specificity of ConA, PNA, VVA, AAL, LTL, and AIL were $75.0 \% / 95.8 \%, 100.0 \% / 64.7 \%, 75.0 \% / 58.3 \%$, $100.0 \% / 72.9 \%, 87.5 \% / 79.2 \%$, and $68.8 \% / 83.3 \%$, separately, in the ICH patients. Our results indicated that $\alpha-M a n / \alpha-G l c$, Gal $\beta 3$ GalNAc, GalNAc, Fuc $\alpha 6$ GlcNAc, $\alpha$-Fuc, and Gal $\beta 3$ GalNAc-Ser might be promising biomarkers to support clinical decision making in diagnosis at the super-acute phase of $\mathrm{ICH}$.

\section{Correlation analysis of neuroinflammatory cytokines with serum glycans}

We compared both the serum concentrations of two

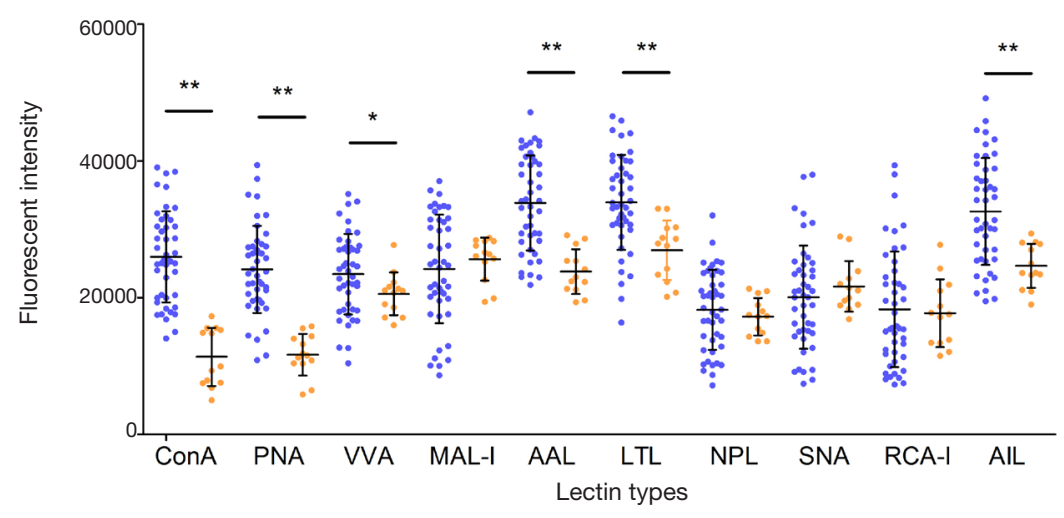

Figure 3 Quantification comparison of 10 kinds of lectin-related serum glycans between 48 ICH patients and 16 healthy controls. The serum concentration of ConA ( $\alpha-M a n / \alpha-G l c)$, PNA (Galp3GalNAc), VVA (GalNAc), AAL (Fuc $\alpha 6$ GlcNAc), LTL ( $\alpha$-Fuc), and AIL (Galß3GalNAc-Ser/Thr) were up-regulated in the ICH patients in comparison with that in the healthy subjects. Spots in indigo: cases; Spots in orange: controls. * $\mathrm{P}<0.05 ;{ }^{* *}, \mathrm{P}<0.001$. ICH, intracerebral hemorrhage. 


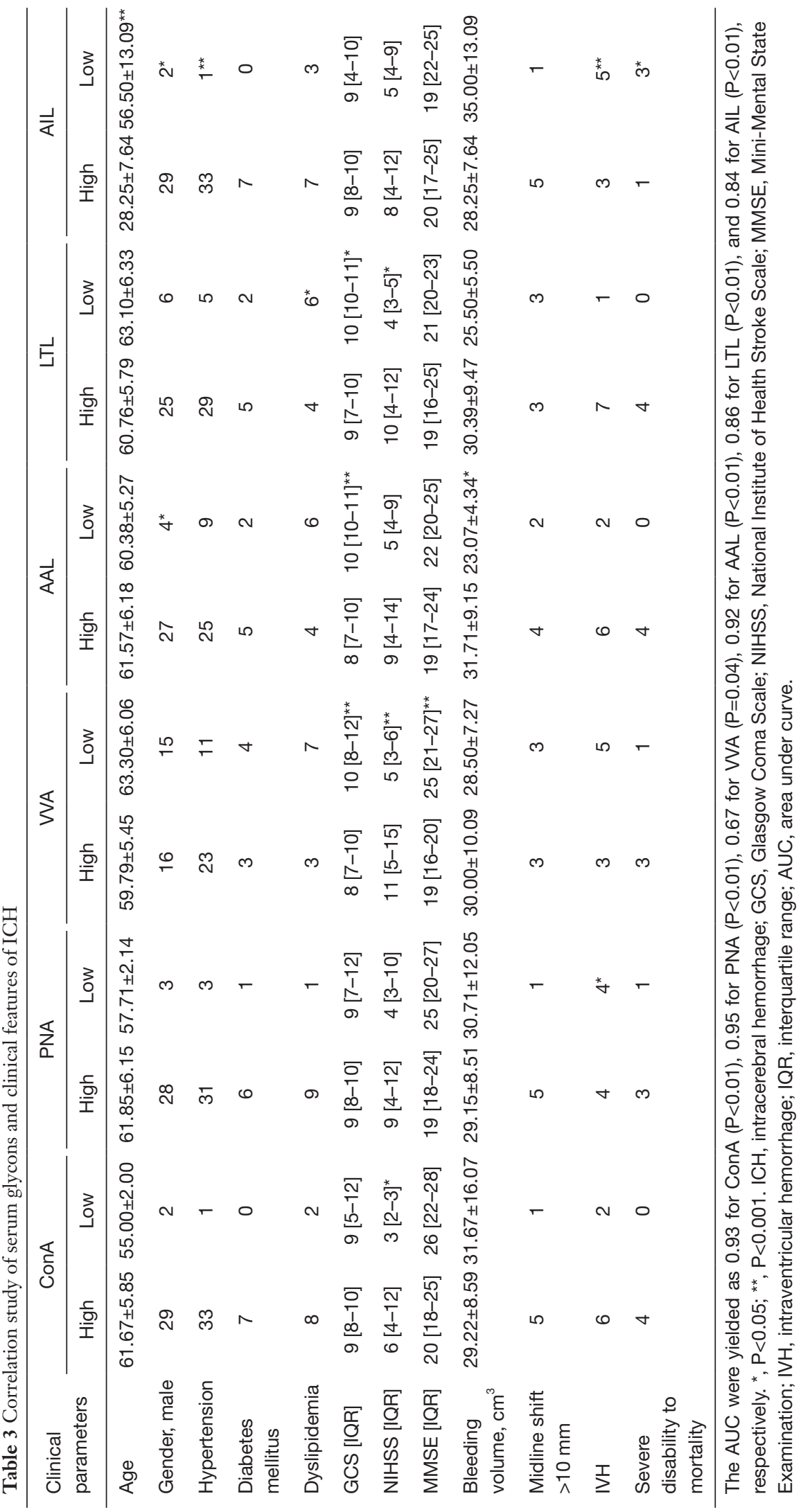


neuroinflammatory cytokines, TNF- $\alpha$ and IL-10, with the serum levels of glycans in ICH. As shown in Figures S2,S3, the serum concentration of TNF- $\alpha$ positively correlated with the serum level of ConA $(r=0.44, \mathrm{P}<0.01)$, and the concentration of serum IL-10 positively correlated with those of ConA $(\mathrm{r}=0.70, \mathrm{P}<0.01)$, PNA $(\mathrm{r}=0.32, \mathrm{P}=0.02)$, VVA $(r=0.40, P<0.01)$, and MAL $(r=0.29, P=0.04)$, respectively.

\section{Discussion}

A variety of glycans or their derivates have been clinically applied as potential diagnostic disease biomarkers. The fucosylated fraction of alpha-fetoprotein (AFP) is a valuable diagnostic biomarker for hepatocellular carcinoma with higher sensitivity and specificity than that of AFP (14). $\mathrm{N}$-glycosylation patterns of multiple plasma proteins revealed potential diagnostic and prognostic values for chronic obstructive pulmonary disease (15), diabetes (16), gastrointestinal, and liver diseases (17).

Similarly, there is increasing evidence showing the obvious effects of glycosylation on neurovascular pathways (18-20). It was reported that down-regulation of CD147 glycosylation was a promising target for neurovascularunit repair in cases of recombinant tissue plasminogen activator (rt-PA)-induced hemorrhagic transformation (21). Other studies interpreted indivisible relations between the glycosylation and IS. Liu et al. found that low levels of galactosylation and sialylation, as well as high levels of bisecting GlcNAc, might be risk factors in IS in comparison with both cerebral arterial stenosis (CAS) patients and healthy subjects (10). However, few investigations relevant to glycans in ICH are available. It was reported that high levels of HbA1c, glycosylated hemoglobin, were an independent prediction indicator for ICH (22). O-GlcNAc modification of cyclin-dependent kinase 5 (CDK5) repressed neural apoptosis through the $\mathrm{p} 53$ pathway in $\mathrm{ICH}$ animal models. These data provide a better understanding of how glycosylation contributes to pivotal neuronal functions in ICH (23). Investigations regarding the effect of glycosylation on stroke patients might provide new insights into the diagnosis and prognosis of hemorrhagic stroke.

In this study, we explored the potential value of serum glycomics' expression for both diagnosis and prognosis in ICH patients using a biochip-based platform. Interestingly, we found that the expressions of glycans such as $\alpha$-Man/ $\alpha$-Glc, Gal $\beta 3$ GalNAc, GalNAc, Fuc $\alpha 6$ GlcNAc, $\alpha$-Fuc, and Gal $\beta 3$ GalNAc-Ser/Thr, were significantly increased in the acute phase of ICH patients compared to those in the healthy controls. We compared ICH patients' clinical diagnostic parameters with six increased serum glycans and found that $\alpha-M a n / \alpha-G l c$ (ConA) exhibited the best specificity. The glycans showing the highest sensitivity were Fuca6GlcNAc (AAL) and Gal $\beta 3$ GalNAc (PNA). Furthermore, in the clinicopathological analysis, we found $\alpha-M a n / \alpha$-Glc (ConA), GalNAc (VVA), and $\alpha$-Fuc (LTL) manifested significantly interpretable values in assessing the recovery of neural function or/and cognitive function. It is worthy to note that $\alpha-M a n / \alpha-G l c$ (ConA) may be a potential biomarker with diagnostic and prognostic value in $\mathrm{ICH}$.

Additional evidence indicated that inflammatory responses might be associated with the dual effect of aggravating brain damage and promoting brain recovery in the phase of secondary brain injury after stroke $(24,25)$. Numerous cytokines and chemokines are involved in ICH's pathological processes and other brain damages. Among these, TNF- $\alpha$ and IL-10 have been widely investigated and considered inflammatory biomarkers and potential therapeutic targets for stroke (26-28). It is well known that TNF- $\alpha$ (pro-inflammatory and neurotoxic) and IL-10 (anti-inflammatory and neuroprotective) display reverse functions in neuroinflammatory processes (25), which were significantly correlated with changes to serum glycans $(29,30)$. In this study, we carried out a correlation efficiency analysis for evaluating the serum expressions of glycans with serum IL-10 and TNF- $\alpha$ levels in ICH patients. We found that the serum concentration of TNF- $\alpha$ was significantly correlated with serum levels of ConA $(r=0.44, \mathrm{P}<0.01)$, and the concentration of serum IL-10 was significantly correlated with those of ConA $(r=0.70, P<0.01)$, PNA $(\mathrm{r}=0.32, \mathrm{P}=0.02)$, VVA $(\mathrm{r}=0.40, \mathrm{P}<0.01)$, and MAL $(\mathrm{r}=0.29$, $\mathrm{P}=0.04)$, respectively. It was reported that $\mathrm{N}$-glycans could play a switch role between pro- and anti-inflammatory $\operatorname{IgG}$ functionality via attaching to the conserved asparagine 297 in the fragment crystallizable $(\mathrm{Fc})$ region (31).

Furthermore, envelope proteins of hepatitis $\mathrm{C}$ virus (HCV) integrated with glycans could protect the conserved immunogenic epitopes from neutralizing antibodies in $\mathrm{HCV}$ infected patients (29). Our data further indicated that glycans could be involved in the anti- or/and proinflammatory reactions in ICH. Collectively, this evidence alerts us to investigate the association between glycomics and serum cytokines in ICH. 
Biochip techniques are a promising method for the fast detection of multiple molecules, including proteins and nucleotides. We introduced a novel lectin-based biochip methodology for detecting glycans from clinical serum samples of patients with ICH. The biochip's sensitivity, specificity, and biocompatibility proved to be potentially applicable in both laboratory and clinical utilization. We suggest that the biochip technique might become a suitable early detection platform due to its remarkable advantages of easy handling, timeliness, and minimal sample requirement. Also, the high throughput of data acquirement allows a preliminary screening with a panel of predictive molecules for disease in a vast cohort of patients. It is also essential to further explore whether glycans could effectively predict the onset of ICH in patient groups with high risks, such as hypertension and vascular malformation.

There were several limitations to this study. Firstly, the sample panel was relatively small, and the study was conducted at a single clinical center only. Secondly, only the serum levels of glycans were measured instead of identifying derived glycan chains or glycoproteins. Finally, a time course of sampling should have been designed to trace the changes in serum glycans from the admission point through the ICH patients' treatment course. In further studies, we will enlarge the study spectrum to evaluate the diagnostic and prognostic roles of serum glycans in augmented ICH samples and analyze the potential relationships between glycomics and glycoproteomics in a larger ICH cohort.

\section{Conclusions}

In summary, we established a lectin-biochip based platform for the fast detection of serum glycomics in ICH patients. To our knowledge, this is the first study investigating serum glycomics as potential biomarkers. Glycans may become potential diagnostic biomarkers in the super-acute phase of ICH and display predictable and prognostic roles in evaluating the recovery of neural function and cognitive function in $\mathrm{ICH}$.

\section{Acknowledgments}

Funding: This study was funded by the Translational Medicine Research Program of Anhui Province of China (grant No. 2017zhyx37), the National Natural Science Foundation of China (grant No. 81901238) and University Natural Science Research Project of Anhui Province (grant
No. KJ2019A0248).

\section{Footnote}

Reporting Checklist: The authors have completed the STARD reporting checklist. Available at http://dx.doi.org/10.21037/ atm-20-7315

Data Sharing Statement: Available at http://dx.doi. org/10.21037/atm-20-7315

Conflicts of Interest: All authors have completed the ICMJE uniform disclosure form (available at http://dx.doi. org/10.21037/atm-20-7315). The authors have no conflicts of interest to declare.

Ethical Statement: The authors are accountable for all aspects of the work in ensuring that questions related to the accuracy or integrity of any part of the work are appropriately investigated and resolved. The study was conducted following the Declaration of Helsinki (as revised in 2013) and was approved by the regional ethics board of the First Affiliated Hospital of Anhui Medical University (No. P2020-12-07). Informed consent was obtained from all individual participants.

Open Access Statement: This is an Open Access article distributed in accordance with the Creative Commons Attribution-NonCommercial-NoDerivs 4.0 International License (CC BY-NC-ND 4.0), which permits the noncommercial replication and distribution of the article with the strict proviso that no changes or edits are made and the original work is properly cited (including links to both the formal publication through the relevant DOI and the license). See: https://creativecommons.org/licenses/by-nc-nd/4.0/.

\section{References}

1. Rajashekar D, Liang JW. Intracerebral Hemorrhage. In: StatPearls [Internet]. Treasure Island: StatPearls Publishing, 2020.

2. Virani SS, Alonso A, Benjamin EJ, et al. Heart disease and stroke statistics-2020 update: a report from the American Heart Association. Circulation 2020;141:e139-596.

3. Hemphill JC 3rd, Greenberg SM, Anderson CS, et al. Guidelines for the management of spontaneous intracerebral hemorrhage: a guideline for healthcare 
professionals from the American Heart Association/ American Stroke Association. Stroke 2015;46:2032-60.

4. Howe MD, Zhu L, Sansing LH, et al. Serum markers of blood-brain barrier remodeling and fibrosis as predictors of etiology and clinicoradiologic outcome in intracerebral hemorrhage. Front Neurol 2018;9:746.

5. Chen W, Wang X, Liu F, et al. The predictive role of postoperative neutrophil to lymphocyte ratio for 30-day mortality after intracerebral hematoma evacuation. World Neurosurg 2020;134:e631-5.

6. Ferrete-Araujo AM, Rodriguez-Rodriguez A, EgeaGuerrero JJ, et al. Brain injury biomarker behavior in spontaneous intracerebral hemorrhage. World Neurosurg 2019;132:e496-505.

7. Liu R, Cao S, Hua Y, et al. CD163 expression in neurons after experimental intracerebral hemorrhage. Stroke 2017;48:1369-75.

8. Kumar A, Misra S, Yadav AK, et al. Role of glial fibrillary acidic protein as a biomarker in differentiating intracerebral haemorrhage from ischaemic stroke and stroke mimics: a meta-analysis. Biomarkers 2020;25:1-8.

9. Zoldoš V, Horvat T, Lauc G. Glycomics meets genomics, epigenomics and other high throughput omics for system biology studies. Curr Opin Chem Biol 2013;17:34-40.

10. Liu D, Zhao Z, Wang A, et al. Ischemic stroke is associated with the pro-inflammatory potential of N-glycosylated immunoglobulin G. J Neuroinflammation 2018;15:123.

11. Deng W, Cao J, Chen L, et al. Plasma glycoproteomic study of therapeutic hypothermia reveals novel markers predicting neurologic outcome post-cardiac arrest. Transl Stroke Res 2018;9:64-73.

12. Ye L, Huang NL, Ma XL, et al. Establishment of N-succinimidyl 4-(maleimidomethyl) cyclohexanecarboxylate (SMCC) modified biochip enabling concurrent detection of serum infectious antibodies in neuroborreliosis. Biosens Bioelectron 2016;78:404-10.

13. Gao Y, Li SG, Liu Q, et al. Establishment of a 1, 4, 7, 10-tetraazacyclododecane-1,4,7,10-tetraacetic acid monoN-hydroxysuccinimide ester (DOTA-NHS-ester) based lectin microarray for efficiently detecting serum glycans in gastric cancers. Anal Biochem 2020;597:113686.

14. Hart GW, Copeland RJ. Glycomics hits the big time. Cell 2010;143:672-6.

15. Pavić T, Dilber D, Kifer D, et al. N-glycosylation patterns of plasma proteins and immunoglobulin $\mathrm{G}$ in chronic obstructive pulmonary disease. J Transl Med 2018;16:323.

16. Rudman N, Gornik O, Lauc G. Altered N-glycosylation profiles as potential biomarkers and drug targets in diabetes. FEBS Lett 2019;593:1598-615.

17. Verhelst X, Dias AM, Colombel JF, et al. Protein glycosylation as a diagnostic and prognostic marker of chronic inflammatory gastrointestinal and liver diseases. Gastroenterology 2020;158:95-110.

18. Ohtsubo K, Marth JD. Glycosylation in cellular mechanisms of health and disease. Cell 2006;126:855-67.

19. Hwang H, Zhang J, Chung KA, et al. Glycoproteomics in neurodegenerative diseases. Mass Spectrom Rev 2010;29:79-125.

20. Iqbal S, Ghanimi Fard M, Everest-Dass A, et al. Understanding cellular glycan surfaces in the central nervous system. Biochem Soc Trans 2019;47:89-100.

21. Xie Y, Wang Y, Ding H, et al. Highly glycosylated CD147 promotes hemorrhagic transformation after rtPA treatment in diabetes: a novel therapeutic target? J Neuroinflammation 2019;16:72.

22. Chen YS, Lian LF, Xu YH, et al. Association of glycosylated hemoglobin level at admission with outcomes of intracerebral hemorrhage patients. Zhonghua Liu Xing Bing Xue Za Zhi 2019;40:1445-9.

23. Ning X, Tao T, Shen J, et al. The O-GlcNAc modification of CDK5 involved in neuronal apoptosis following in vitro intracerebral hemorrhage. Cell Mol Neurobiol 2017;37:527-36.

24. Jin R, Liu L, Zhang S, et al. Role of inflammation and its mediators in acute ischemic stroke. J Cardiovasc Transl Res 2013;6:834-51.

25. Ye L, Gao L, Cheng H. Inflammatory profiles of the interleukin family and network in cerebral hemorrhage. Cell Mol Neurobiol 2018;38:1321-33.

26. Nakamura K, Shichita T. Cellular and molecular mechanisms of sterile inflammation in ischaemic stroke. J Biochem 2019;165:459-64.

27. Esenwa CC, Elkind MS. Inflammatory risk factors, biomarkers and associated therapy in ischaemic stroke. Nat Rev Neurol 2016;12:594-604.

28. Lambertsen KL, Biber K, Finsen B. Inflammatory cytokines in experimental and human stroke. J Cereb Blood Flow Metab 2012;32:1677-98.

29. Lavie M, Hanoulle X, Dubuisson J. Glycan shielding and modulation of hepatitis $\mathrm{C}$ virus neutralizing antibodies. Front Immunol 2018;9:910. 
30. Baum LG, Cobb BA. The direct and indirect effects of glycans on immune function. Glycobiology 2017;27:619-24.

31. Alter G, Ottenhoff THM, Joosten SA. Antibody

Cite this article as: Ye L, Fang YS, Li XX, Gao Y, Liu SS, Chen Q, Wu Q, Cheng HW, Du WD. A simple lectin-based biochip might display the potential clinical value of glycomics in patients with spontaneous intracerebral hemorrhage. Ann Transl Med 2021;9(7):544. doi: 10.21037/atm-20-7315 glycosylation in inflammation, disease and vaccination. Semin Immunol 2018;39:102-10. 


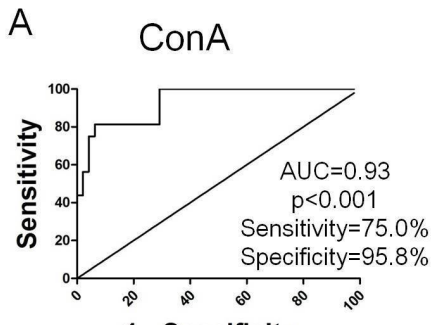

1 - Specificity

D

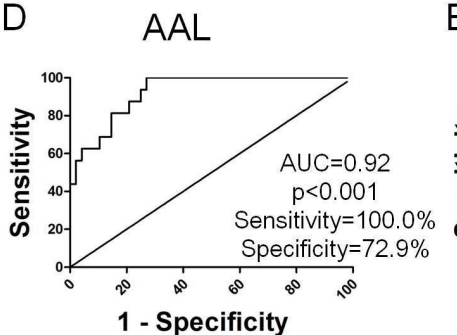

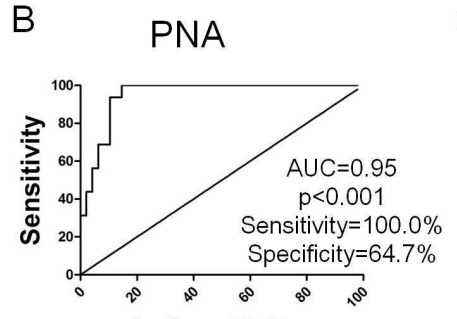

1 - Specificity

E

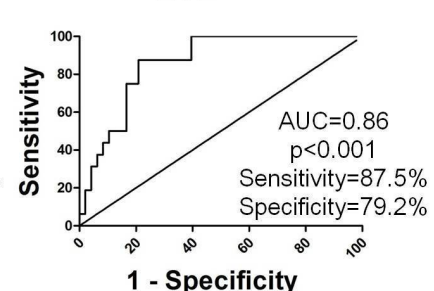

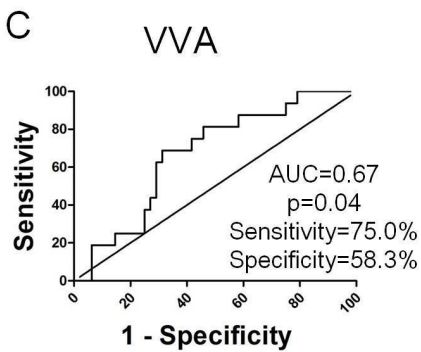

F

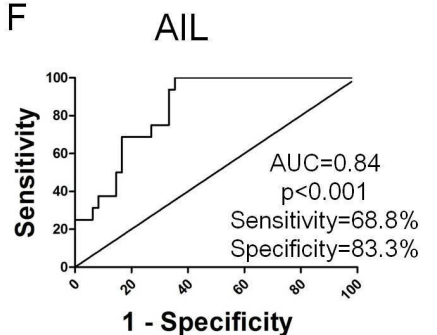

Figure S1 Diagnostic values for six lectin-related glycans in ICH patients using ROC curve. The AUC were yielded as 0.93 for ConA $(\mathrm{P}<0.01)$ (A), 0.95 for PNA ( $\mathrm{P}<0.01)$ (B), 0.67 for VVA $(\mathrm{P}=0.04)(\mathrm{C}), 0.92$ for AAL $(\mathrm{P}<0.01)(\mathrm{D}), 0.86$ for $\mathrm{LTL}(\mathrm{P}<0.01)(\mathrm{E})$, and 0.84 for AIL $(\mathrm{P}<0.01)$ (F), respectively. ICH, intracerebral hemorrhage; ROC, receiver operating characteristic; AUC, area under curve.

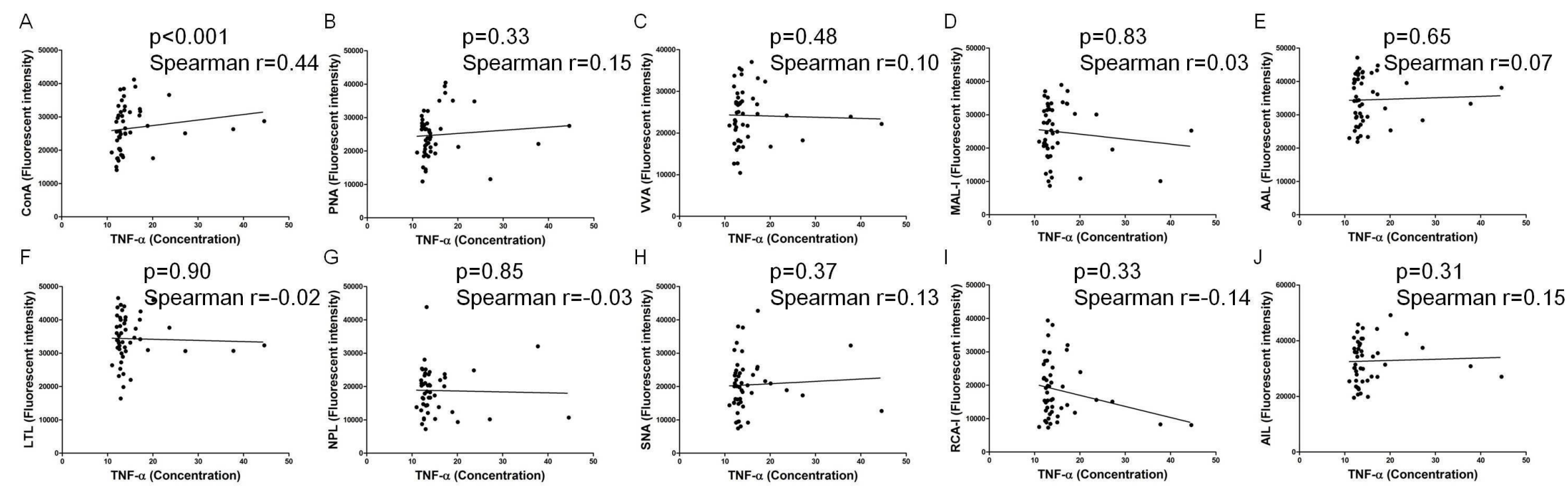

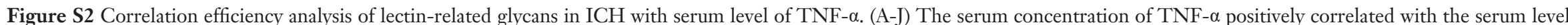
of ConA ( $\mathrm{r}=0.44, \mathrm{P}<0.01)$. ICH, intracerebral hemorrhage. 

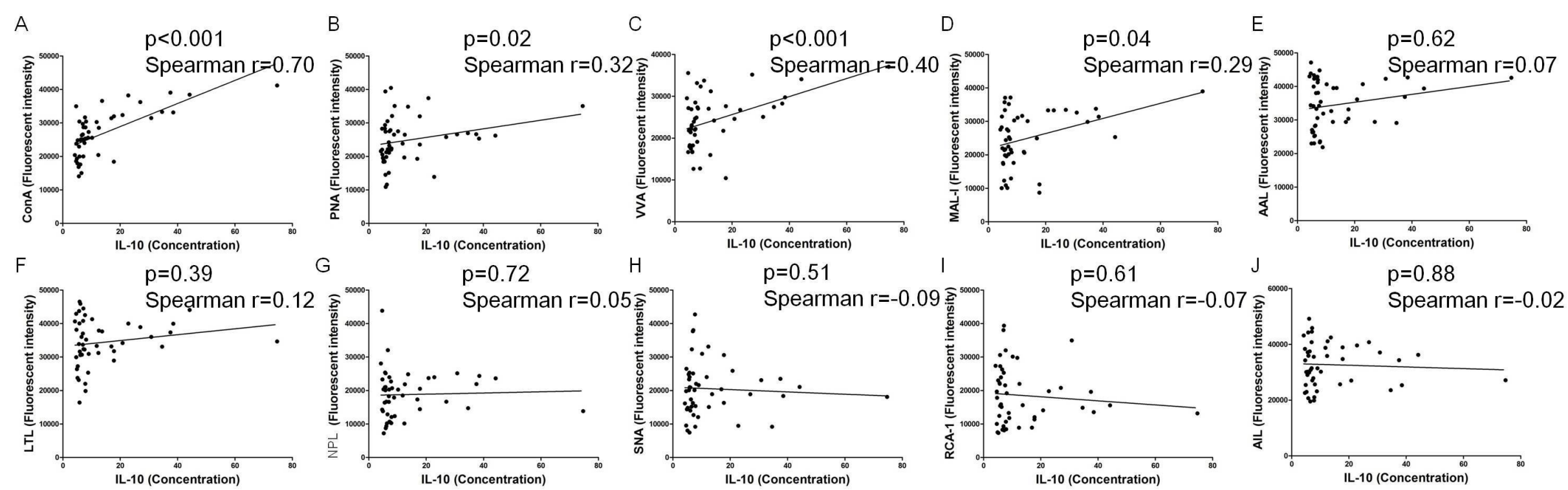

Figure S3 Correlation efficiency analysis of lectin-related glycans in ICH with serum level of IL-10. (A-J) The concentration of serum IL-10 positively correlated with those of ConA ( $\mathrm{r}=0.70$, $\mathrm{P}<0.01)$, PNA $(\mathrm{r}=0.32, \mathrm{P}=0.02)$, VVA $(\mathrm{r}=0.40, \mathrm{P}<0.01)$, and MAL $(\mathrm{r}=0.29, \mathrm{P}=0.04)$, respectively. $\mathrm{ICH}$, intracerebral hemorrhage. 